\title{
Recording online processes in task-oriented reading with Read\&Answer
}

\author{
Eduardo Vidal-Abarca • Tomás Martinez • \\ Ladislao Salmerón • Raquel Cerdán • Ramiro Gilabert • \\ Laura Gil • Amelia Mañá • Ana C. Llorens • \\ Ricardo Ferris
}

Published online: 18 November 2010

(C) Psychonomic Society, Inc. 2010

\begin{abstract}
We present an application to study task-oriented reading processes called Read\&Answer. The application mimics paper-and-pencil situations in which a reader interacts with one or more documents to perform a specific task, such as answering questions, writing an essay, or similar activities. Read\&Answer presents documents and questions with a mask. The reader unmasks documents and questions so that only a piece of information is available at a time. This way the entire interaction between the reader and the documents on the task is recorded and can be analyzed. We describe Read\&Answer and present its applications for research and assessment. Finally, we explain two studies that compare readers' performance on Read\&Answer with students' reading times and comprehension levels on a paper-and-pencil task, and on a computer task recorded with eye-tracking. The use of Read\&Answer produced similar comprehension scores, although it changed the pattern of reading times.
\end{abstract}

Keywords Reading comprehension - Reading strategies . Task-oriented reading $\cdot$ Reading processes .

Reading assessment

The study of on-line reading processes has a long history (Just \& Carpenter, 1980; Rayner, 1998). It has mainly been conducted using the eye movement technique, although some other techniques based on reading time have also been employed (see Rouet \& Passerault, 1999, for a

E. Vidal-Abarca $(\bowtie) \cdot$ T. Martinez $\cdot$ L. Salmerón $\cdot$ R. Cerdán $\cdot$

R. Gilabert $\cdot$ L. Gil · A. Mañá $\cdot$ A. C. Llorens $\cdot$ R. Ferris

University of Valencia,

Avda. Blasco Ibáñez, 21,

46010, Valencia, Spain

e-mail: vidala@uv.es review). Although these techniques have been used to study reading at many levels (e.g. syllable, word, sentence, etc.), our main interest is in methodologies and techniques that focus on the discourse level.

Eye movement research on reading at the discourse level has focused on how readers process a text. ${ }^{1}$ This research has considerably increased our knowledge about reading strategies (e.g., Duggan \& Payne, 2009; Hyönä, Lorch, \& Kaakinen, 2002; Kaakinen \& Hyönä, 2005; Kaakinen, Hyönä, \& Keenan, 2003). However, the use of the eye movement technique has been limited by many factors. Just to mention a few, eye trackers are expensive, not particularly durable, and rarely portable. Their use requires frequent recalibration, data analysis is time-consuming and tedious, and participants have to be tested individually. To overcome these limitations and to work with a tool specifically designed to study task-oriented reading processes at the discourse level, we developed Read\&Answer. Read\&Answer is free software that runs on almost any Windows computer and can be run from a USB pen. It does not require any calibration, it can be self-learned after a 1-h tutorial, and it allows concurrent evaluation of several students on task-oriented reading activities.

This paper has two goals. First, we describe Read\&Answer and its possibilities for research purposes and assessment. Second, we explain two studies conducted to validate the tool. These goals correspond to two main sections of this paper. However, before describing Read\&Answer, we briefly explain the task-oriented reading approach to provide a

\footnotetext{
${ }^{1}$ Following the terminology of the OECD (2002), we use the word text to refer to any document that includes not only textual information made up of sentences grouped into paragraphs, but also graphical or pictorial information, such as a graph, a diagram, a table, and so on.
} 
global picture of the situations for which Read\&Answer was specifically designed. Then, we introduce the tools to record the reading process at the discourse level that inspired the design of our tool, and we describe the basic necessary elements of a tool to record reading behavior in task-oriented reading.

\section{Task-oriented reading approach}

Task-oriented reading refers to situations in which a reader reads one or more texts while knowing in advance that $\mathrm{s} / \mathrm{he}$ has to perform a task for which the texts are a crucial and available source of information. The task can involve answering some questions from a text (Cerdán, VidalAbarca, Martínez, Gilabert, \& Gil, 2009), writing an essay based on information from several texts (Cerdán \& VidalAbarca, 2008), taking notes from several texts while writing an essay (Gil, Vidal-Abarca, \& Martínez, 2008), or any other task requiring the use of one or more texts to give some sort of written answer while having the text information available to search (Gil, Braten, Vidal-Abarca, \& Stromso, in press). This situation requires the reader to interact with the text in a special way, going back and forth from the text to the task and vice versa.

There is evidence that this interaction puts specific demands on the reader beyond basic comprehension processes at the discourse level (see Rouet, 2006 for a broad discussion of this issue). For instance, Ozuru, Best, Bell, Witherspoon, and McNamara (2007) found that prior background knowledge was not predictive of comprehension scores when readers had to answer questions from a text that was available while answering the question, whereas it was when the students had to answer the questions without referring back to the text. Mañá, VidalAbarca, Dominguez, Gil, and Cerdán (2009) found that the ability to monitor the comprehension of the task and regulate the interaction with the text explained a significant percentage of variance in comprehension in a task-oriented reading situation, beyond the explanation provided by a general measure of reading comprehension skills. VidalAbarca, Mañá, and Gil (in press) found that monitoring the decision to search the text in a task-oriented reading situation did not depend on general comprehension skills, but rather on some variables related to the interaction between the reader and the text. All these results can be explained by the fact that task-oriented reading requires students to make more decisions than just reading a text. For instance, readers have to decide what to read first, the text or the task. They also have to make a decision about what part of the text is relevant for this specific task, and what part may be skipped or read more superficially. Readers also have to decide when searching is not necessary anymore because either they believe they already know the answer or the task is completed.

Task-oriented reading activities such as the ones described above are common in educational settings and many other situations. Moreover, recent definitions of reading literacy developed by reading literacy experts emphasize the type of interaction between the reader and the text on different sorts of activities referred to here as task-oriented reading (Snow, Snow and RAND Reading Study Group 2002; OECD, 2002). Thus, a tool like Read\&Answer, specifically designed to study taskoriented reading activities, will help to shed light on how readers interact with a text on those sorts of tasks. It must be highlighted here that Read\&Answer is limited to studying paper-and-pencil-like task-oriented reading activities, although presented on a computer in a special way so that interaction processes can be recorded.

\section{Features of a tool to record task-oriented reading processes}

In this section, we first examine basic features of research tools based on masking procedures that have tried to mimic the eye movement technique. Then, we will describe some conclusions about the design of a tool to record taskoriented reading processes that have directly inspired the development of Read\&Answer.

Tools based on masking procedures Some tools mimicking the eye movement approach at the discourse level have been developed. They all have in common the use of a sort of moving window technique (McConkie \& Rayner, 1975). They present the reader with a text to be read on a computer screen. The text is visible but perturbed except in the area the reader is actually reading. Readers are free to move through the text whenever and wherever they wish by clicking or moving on a new area with the mouse. When they proceed to a new area, the previous part is masked, and the new one is shown. It is also possible to go back to a previously read area using the mouse. The computer records the reading path as well as the time spent on each predefined area in milliseconds. Thus, it is possible to record students' on-line reading behavior in conditions similar to natural reading.

Therefore, common characteristics of these tools, in comparison to eye tracker devices, are the following. First, the required equipment is very cheap, i.e., only a personal computer with the specific software. Second, they do not need any sort of careful calibration in advance like the eye tracker does, so the preparation needed to display the stimuli is very simple. Third, data obtained are accurate, independently of students' head movements during the 
reading session. Fourth, the procedure is non-intrusive. Fifth, there is no interference for participants wearing glasses. Next, we present two tools that use some sort of masking procedure and have inspired the development of Read\&Answer.

The first tool, called Select-the-Text, was developed to study the reading strategies students use to move through a text (Goldman \& Saul, 1990a). Although this tool is no longer in use, it has inspired the development of Read\& Answer, and we have retained some of its features. Selectthe-Text presented readers with a full screen of text. The entire text was masked, except the sentence currently selected by the reader, and the white spaces denoted sentence boundaries. Readers unmasked a sentence by clicking on it; when they unmasked another sentence, the first sentence was masked again. Thus, only one sentence at a time was visible, but the visual features of the text, e.g., paragraph indentation, length of the passage, and position of the sentence in the paragraph and passage, were visible to the reader. The researcher decided on the size of the masked text based on research purposes, but it varied within a text according to the sentence's length. The tool provided an output file that contained raw data with the time during which each sentence was exposed in milliseconds, and a summary table. Goldman and colleagues used Select-the-Text to examine reading strategies of readers of different ages (Coté, Goldman, \& Saul, 1998; Goldman \& Saul, 1990b). Rouet, VidalAbarca, Bert-Erboul, and Millogo (2001) have also used Select-the-Text to investigate the effects of information search tasks on university students' comprehension of a scientific text.

Jansen, Blackwell, and Marriot (2003) developed another tool called Restricted Focus Viewer (RFV). RFV is a computer program that takes a visual stimulus, blurs it, and displays it on a computer monitor, allowing the participant to see only a small region of the stimulus in focus at any time. The region in focus can be moved using the computer mouse. RFV records what the participant is focusing on at any point in time, and the data can be played back using Replayer, a companion program that can replay the way a participant moved the focus window over the stimuli. The size of the focus windows, which is fixed throughout the stimuli for a specific presentation, is set by the researcher following two criteria: (1) the blurred image of the stimulus should reveal enough information to allow a participant to successfully navigate from one region of interest to another; (2) participants should not be able to identify different syntactic elements without moving the focus window over them. Jansen et al. (2003) have compared RFV and the eye movement technique on a simple reading task (experiment 1 , examining algebraic expressions to answer some questions) and a complex task (experiment 2, examining some diagrams of pulley systems and answering questions about the motion of their components). They found no differences in performance and similar scan-paths when comparing RFV and eye movements, although students spent significantly more time when they performed the task with RFV.

In a follow-up study, Bednarik and Tukiainen (2007) compared RFV technology and the eye movement technique in a more precise way on a more complex task. They compared how expert and novice programmers detected some errors in the source code while moving through several screen windows (e.g., the source code, the debugger output and some other representations) displayed simultaneously on a computer screen, a natural task for programmers. Programmers performed some tasks with RFV (on-RFV) and others without the blurring RFV procedure (off-RFV), and their eye movements were recorded in both cases. RFV did not affect the detection of errors, but it introduced some changes in the strategies used by the experts (e.g., more switches between windows in on-RFV than in off-RFV). There was no difference for the novice programmers. As in the previous study, it also took the participants longer to perform the task with RFV than without RFV.

Conclusions about the design of a tool to record taskoriented reading processes Basic common features of Select-the-Text and RFV have been retained in the development of Read\&Answer. No doubt, the most important one is the unmasking/masking procedure, which allows the researcher to record the reader's actions when $\mathrm{s} / \mathrm{he}$ is reading the text or interacting between the text and the task. Other common features, such as the use of the mouse to move from one area of interest (AOI) to another, are also relevant and have been incorporated into Read\&Answer. In addition, some specific features of each masking tool have also been retained.

An important feature of Select-the-text, which is not available in RFV, is that the size of the window within a text is not fixed, but can be set depending on research purposes. This feature is important because text sentences vary in length. Beyond this advantage, flexibility in window size is also crucial for a tool that records the reader's behavior in a task-oriented-reading situation. In this type of situation, it is important to associate a specific sentence or a group of sentences with a specific task. For instance, when answering a question while having a text available, it is important to know whether or not the reader uses the relevant information to answer that question. Thus, the association between a question and relevant text information to answer that question is crucial for analyzing the reader's behavior when answering. This association is clearer if the researcher can define areas of interest (AOIs) according to an analysis of the questions. Otherwise, the relationship between the task (e.g., the question) and the text is more obscure. In addition, the researcher may be 
interested in testing specific hypotheses that require controlling the AOI the reader has available (e.g., to test the role of a heading in the task). However, an important limitation of Select-the-text is that it is only possible to present plain text. Thus, Select-the-Text cannot display any typographical features or, more importantly, any graphical information (e.g., a graph or a diagram).

An advantage of Select-the-Text is a limitation of RFV, and vice versa. RFV is inflexible regarding the size of the presentation unit within a text, but it allows the presentation of any kind of information, whether textual or graphical. Therefore, we considered that Read\&Answer should retain the two main advantages mentioned above, i.e., flexibility in the unit of presentation and the possibility of presenting any kind of information.

In addition to common and specific characteristics of Select-the-Text and RFV, Read\&Answer needs to incorporate new features that are specific to task-oriented reading situations. The most important one is the possibility of reproducing the basic structure of any task-oriented reading scenario in which the reader reads one or more texts and then performs a specific task, such as answering some questions or writing an essay. This basic structure was implemented in a previous and simpler version of Read\&Answer (see for example Cerdán \& Vidal-Abarca, 2008) by including a text screen for reading the text and a question screen for performing a task, with a simple interface that allows the reader to move from one to the other and vice versa. In that version we retained the unmasking/masking procedure, so that the researcher could know what action the reader was performing at any time and for how long.

To accommodate task-oriented reading scenarios, the previous and simpler version of Read\&Answer presented texts in a page-like layout, so that the location of a piece of information on a page was fixed, mimicking a paper text. This is important when the reader is allowed to search the text to perform the task. However, this previous Read\&Answer version had some important limitations. It was only able to present plain text. Additionally, it was designed to present open-ended questions, but presenting any other question format (e.g., multiple-choice) required important adaptations. To overcome these limitations, we developed the new version of Read\&Answer described in the following section.

\section{Description of Read\&Answer 2.0}

Read\&Answer 2.0 is an application that enables researchers to develop a wide variety of experiments that can be implemented with masked or unmasked presentation of text or pictures, and different kinds of tasks. Read\&Answer 2.0 allows the recording of on-line readers' actions when they read a text or examine pictures and answer questions about them, providing the sequence of actions (i.e., re-read a piece of text, picture or question) and time spent on each action. In this section we first specify some technical features of the program. Then, we briefly clarify how to design a basic experiment with Read\&Answer 2.0, and we explain how Read\&Answer 2.0 is presented to participants when performing task-oriented reading. We also present complementary software called RADAR (Read\&Answer Data Analysis Resource) that assists researchers with the analysis of the data generated by Read\&Answer 2.0.

Technical features of Read\&Answer 2.0 Read\&Answer 2.0 is a Microsoft Windows (Windows XP, Vista, or Windows7) desktop application, developed on .Net technology (with Microsoft Visual Studio $\subset$ 2008). This application requires the operating system to be updated with the .NET Framework. Other minimum requirements are identical to those of the operating system installed on the computer, and the application requires $2 \mathrm{MB}$ of additional hard-disk space. No serial numbers or license codes are needed.

The program can be downloaded as freeware from the web site of the Comprehension and Learning from Text research group at the University of Valencia $<$ http://sites. google.com/site/psicotext/tecnologia-educativa-educationaltechnology> in a zip file. Spanish and English versions are available. The installation procedure requires the user to have system administrator rights. Users should extract the installer and run the msi file to first check whether .NET Framework 3.5 is installed, and install it if necessary. Afterwards, the Read\&Answer 2.0 application will be installed.

Designing an experiment with Read\&Answer 2.0 Read\& Answer 2.0 is composed of two main modules: Design experiment module and Run Experiment module. When initiating Read\&Answer 2.0, a window appears presenting these two options. In order to design an experiment, the user needs to access the design experiment module and perform two main actions: first, prepare and segment the documents; second, create associated tasks. In order to prepare a text, it has to first be imported in $\mathrm{rtf}$ format page by page. In other words, if a specific text is composed of three pages, the designer needs to prepare three different $\mathrm{rtf}$ documents in advance, each of them corresponding to the visual display of the three pages.

Once the rtf documents have been loaded, the next step is to create the masking regions that participants will be able to uncover while reading. Given that an action is equivalent to the period during which a region is unmasked, it is important to decide the length of the regions according to research needs (e.g., sentence, paragraph level). To create a region, Read\&Answer 2.0 provides a toolbar at the right 
of the screen where the number of specific regions needed for a given page can be inserted a priori. Regions will be associated with colors, which will enable a visual display of the page segmentation. Once the number of regions and associated colors has been created, the user has to specify on the screen the area for each of the regions, by clicking on the text and moving the mouse pointer while maintaining the mouse button pressed. The final result for a page is a visual display of the specific segmentation planned for the experiment (see Fig. 1). Please note in Fig. 1 the visual segmentation of the colored areas corresponding to regions that the student will be able to uncover by clicking on them. Some of these areas adopt the form of a rectangle, but Read\&Answer also makes it possible to incorporate sentences that end or begin in the middle of a specific paragraph.

Read\&Answer 2.0 allows the inclusion of an undetermined number of pages for a given text and an undetermined number of texts. Read\&Answer 2.0 also makes it possible to create tasks, which are composed of one or more questions and a corresponding text. Thus, a question is always a part of a specific task, and each task (and its related questions) is always linked to one specific text. The researcher may create as many tasks as required in each text, by using the features of the question editor. It is an option for the researcher to decide whether or not participants will be able: (1) to modify an answer once they have moved to another question and (2) to refer back to the text to search for answers. Questions may be created in multiple-choice and open-ended formats.
Additionally, Read\&Answer 2.0 allows the researcher to use some advanced possibilities, such as adding some statements before and after the questions, which can be used for different research purposes (see, for example, Vidal-Abarca et al., in press, for two uses of these advanced possibilities: monitoring the comprehension of a question and Judgment of Learning before answering a question). A more complete description of these possibilities can be found in the user's guide included in the downloadable package.

Performing task-oriented-reading with Read\&Answer 2.0 When a specific experiment has been designed, it can be run by accessing the run experiment module after starting the program. Participants are first asked to fill in some relevant profile data that the researcher will have specified in advance when saving the experiment in the design phase. Next, they are presented with the masked text and a navigation toolbar at the right of the screen. To read the text, participants need to click on each region to unmask it (Fig. 2). When a region is accessed and unmasked, the previous one is re-masked. Participants are also able to proceed to the next page or move to a different text by using a navigation toolbar at the right of the screen. The arrow symbol allows participants to go back and forth between the pages of a text, whereas the symbols plus and minus allow them to go back and forth among the different texts (Fig. 2).

To access the question screen, participants have to click on the question mark button in the toolbar at the right of the
Fig. 1 Visual display of a segmented page

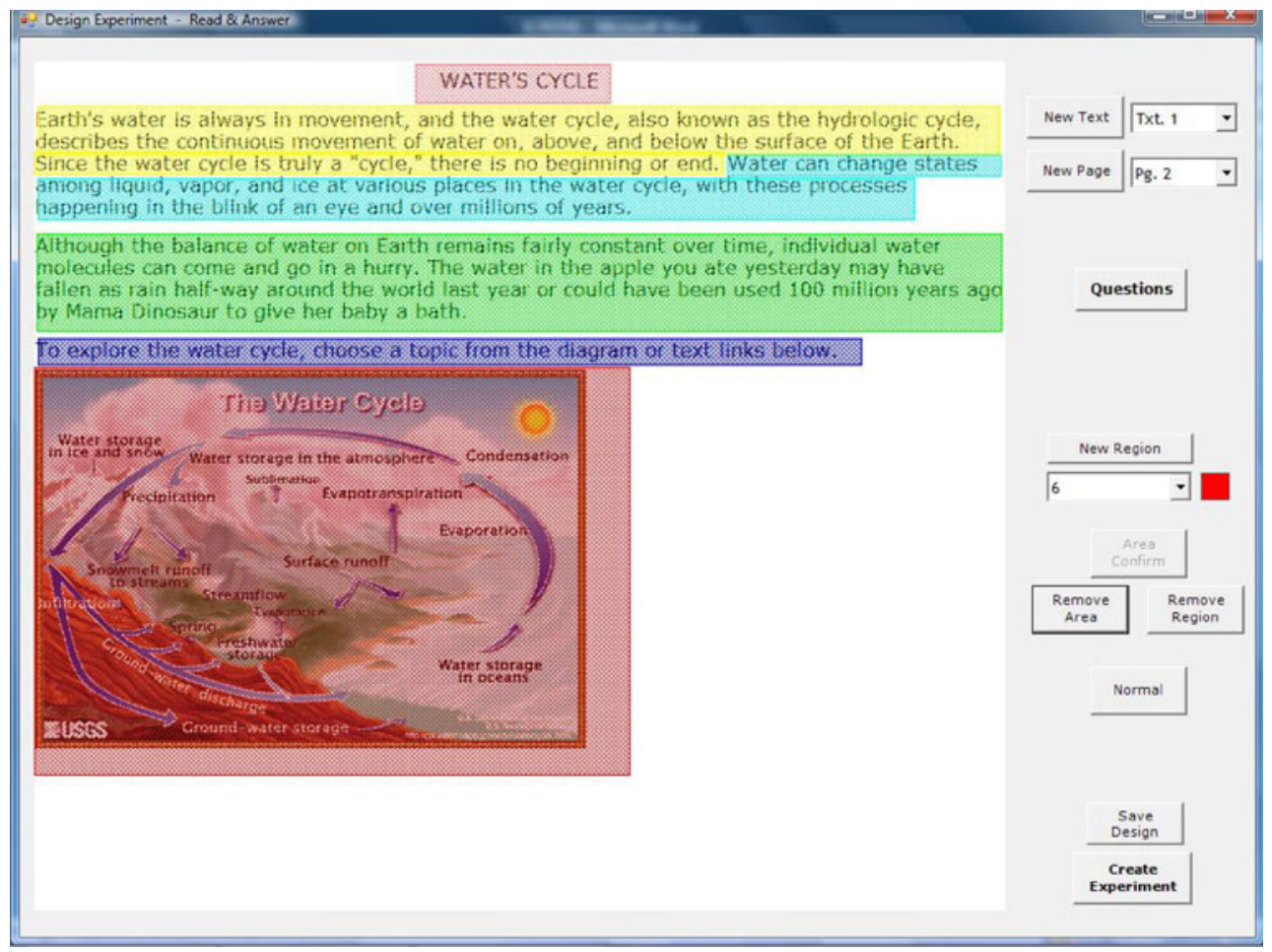


Fig. 2 Masked and unmasked regions of a text and the navigation toolbar

\section{कs}

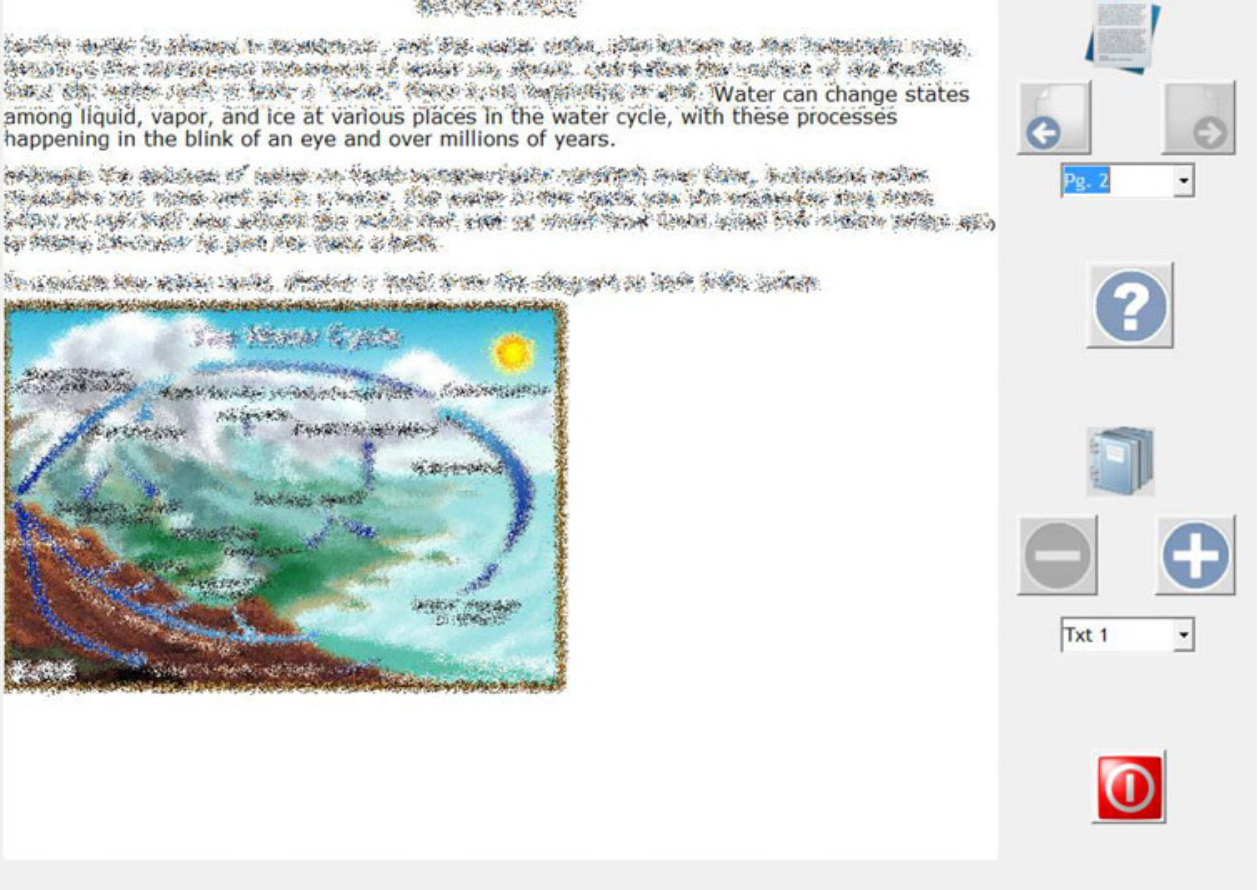

screen (Fig. 2). Then, a new screen appears with the first question or task (Fig. 3). Both the question and the answer will be visible, but not readable. In the case of a multiplechoice format, participants have to unmask either the question or the answer (see Fig. 3). For an open-ended question, a box where participants can write the answer is visible under the question. A simple interface at the bottom of the question screen allows participants to move from one question to the next and go back to the text screen in case this option is available. This way, participants can navigate across the text and the questions. Once participants have completed the task, they may exit the application by returning to the text screen and clicking on the exit button (Fig. 2).

Further information on how to install, design and use Read\&Answer 2.0 is available in the user's guide, which can be downloaded from the authors' research web site.

Output analysis: RADAR Read\&Answer 2.0 provides a text file output for each participant with the sequence of actions performed in the experiment. Every line of this sequence of actions contains either two or three parts: the first part identifies the region of the text or question unmasked by the participant, and the second part shows the duration in milliseconds of the unmasking. When registering the unmasking of questions, there is an additional line in the output showing the answer provided for that question.

This output includes only raw data on every action the participant has performed during the experiment, in three different stages of task-oriented reading: (1) when reading the text before going to question screen; (2) when searching for textual information to answer the questions, if the participant decides that a textual search is needed, and/or if the option to search is available; and (3) when answering the questions. Then, specific variables can be created for further analyses. To facilitate this task a new application called RADAR (Read \& Answer Data Analysis Resource) was developed. RADAR is also a Microsoft Windows desktop application, developed on .Net technology, with features, requirements and installation similar to those required by Read\&Answer 2.0. The program can also be downloaded from our web site.

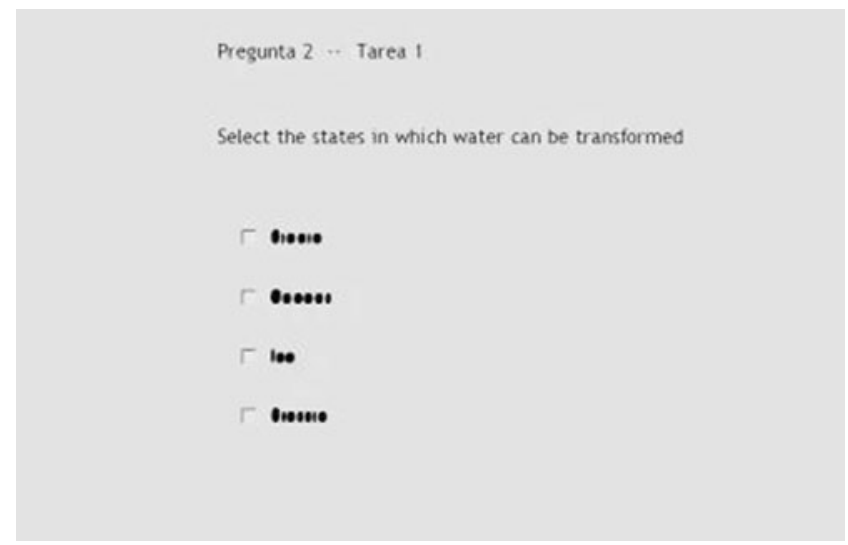

Fig. 3 Question screen of a multiple-choice question 
RADAR generates three summary files per student and a global file that summarizes data for all participants in an experiment. The last file can be exported to a spreadsheet and a statistical program. The process of generating these files is not automatic, and it requires the researcher to complete a series of three steps. First, the researcher selects the file with the definition of a Read\&Answer experiment (.rnw extension). Second, the researcher defines and introduces a number of variables for the analysis. Some variables are obligatory. For instance, the number of words in a region must be introduced so that RADAR can calculate the time/word reading for a region. The correct answer to a multiple-choice question must also be introduced, so that RADAR can score the number of correct answers. Some variables relating pieces of a text and questions are also obligatory. For instance, the researcher must also define text information relevant to answering a specific question. Some other variables are optional. For example, RADAR allows the researcher to define and categorize either text regions or questions. As a third step, once the definition of the experiment has been completed, we can specify the set of sequences we want to analyze with RADAR, and this process will generate the output files to be exportable to data spreadsheets and/or statistical programs.

RADAR output files and variables have been developed as a consequence of several studies (i.e., Cerdan \& VidalAbarca, 2008; Cerdan et al. 2009) conducted with the initial version of Read\&Answer 1.0. In these studies, we worked with the raw sequential data and developed specific measures associated with predetermined research needs.

Thus, depending on the specific hypothesis of a study, it may be interesting to analyze the number of visits to a given region of interest, the reading pace (time per word) in reading a specific region or question, or the number of times the participant has completed a more complex questionanswering behavior (i.e., reading a relevant piece of information and immediately answering the corresponding question). The data provided by Read\&Answer are quite flexible. Given that every action and its corresponding time are registered, the possibilities of creating measures beyond those provided by RADAR to fit specific design needs are quite open.

In general, several measures have been developed associated with specific research needs, all in the general framework of task-oriented reading. These could be grouped into the three stages of analysis we mentioned earlier: first, indicators of the initial reading of a text; second, indicators of the interaction between the text and questions; and, finally, indicators of how participants read and answer questions. In the next subsection, we will briefly report on several studies conducted using Read\&Answer, in order to give the reader further insight into the research possibilities and measures this tool might offer to the researcher interested in a wide variety of task-oriented reading situations.

\section{Uses of Read\&Answer}

As indicated earlier, Read\&Answer is mainly used for research on task-oriented reading. Within this approach, the role of different types of tasks on comprehension processes and learning from texts is an important research topic. For example, Cerdan and Vidal-Abarca (2008) used a previous version of Read\&Answer to compare the effects of two tasks, writing an essay and answering shorter intra-text questions, on integrating information across different texts. They found that writing an essay was more effective at the deep level of comprehension than answering intra-text questions, whereas no difference was apparent at the superficial level of understanding. Read\&Answer provided on-line evidence supporting this conclusion. Students who wrote the essay showed more integrative behavior, consisting of reading a relevant piece of information and then reading another non-consecutive closely-related relevant piece of information, than those who answered intra-text questions. In a follow-up study, Cerdán et al. (2009) found that high-level questions facilitated deep comprehension, but not immediate performance or delayed recall of text, and that both high- and low-level questions differentially affected text-inspection patterns. On-line evidence supporting these conclusions was also found: e.g., high-level questions made students relate separate pieces of text information more often than low-level questions.

The study of individual differences in task-oriented reading is another research topic. Vidal-Abarca and colleagues have investigated how skilled and less-skilled comprehenders differ in monitoring the comprehension of the task and self-regulating their decisions to search the text (Mañá et al., 2009; Vidal-Abarca et al., in press). Read\& Answer provides on-line information about readers' decisions to search the text in order to answer a question, and about how accurate and effective the search is (e.g., the percentage of time reading relevant information or the use of that information to answer a question). It is also possible to study on-line reading strategies when students are free to either read a text first before the questions or read the questions first and then search the text, and these strategies can be related to performance scores (Salmerón, VidalAbarca, Martínez, Mañá, Gil, \& Naumann, 2010).

Theoretical and practical issues regarding the assessment of reading comprehension with a question-answering approach can also be studied with Read\&Answer. For instance, text availability has been suggested to have an impact on assessment of comprehension processes (Ozuru, et al., 2007). These authors concluded that, whereas 
without-text comprehension questions are more suitable for measuring on-line comprehension ability to rapidly access relevant knowledge to recognize familiar content in the text, with-text comprehension questions may be more suitable for assessing readers' ability to engage in strategic processing to fulfill specific reading goals. Ferrer, VidalAbarca, Avila, Mañá, and Llorens (2010) have found online evidence of these strategic differences. It is also possible to develop a test to assess on-line comprehension processes following a task-oriented reading approach. Martinez, Vidal-Abarca, Gil, and Gilabert (2009) have developed a computer-based test that provides information about on-line reading strategies when students read and answer the questions, and when they make decisions to search the text.

Although Read\&Answer has been designed to study reading processes within a task-oriented reading approach, it might also be used to study many theoretical and practical issues on reading at the discourse level. The relevance and perspective effects are some examples. McCrudden and Schraw (2007) define the relevance effect as "the influence of relevance instructions on text learning, text processing time, or both" (p. 115). McCrudden, Magliano, and Schraw (2009) have studied how relevance instructions impact online text processing with a self-paced computer presentation of textual information. Kaakinen, Hyönä, and Keenan (2003) have used the eye-movement technique to study the perspective effect, that is, how an induced perspective (e.g., reading a text describing 4 rare illnesses from a given perspective) makes readers process information relevant to the perspective in a more in-depth way than information irrelevant to this perspective. The analysis of relevance and perspective effects can also be performed with Read\& Answer, as it records the readers' behavior when they process relevant and irrelevant sentences in a text.

Practical issues, such as reading strategies at the discourse level, can also be studied with Read\&Answer. For instance, Hyönä, Lorch, and Kaakinen (2002) have examined the readers' behavior when they read to summarize a long expository passage. These authors found that readers can be classified into four clusters according to their on-line reading behavior (e. g., looks back to previous sentences or reads the headings and sub-headings) and that these clusters are associated with different performance scores. These on-line reading behaviors can also be recorded with Read\&Answer, as it records the readers' actions when processing a text.

In summary, Read\&Answer has been specifically designed to study on-line processes in task-oriented reading. It records the readers' behavior during taskoriented reading activities in three different stages (i.e., first reading a text, inspecting a text to search for information, answering questions), which provide informa- tion about how readers process relevant and irrelevant information, their search processes when performing a task, and their reading strategies. Read\&Answer can also be used as an alternative to the eye movement technique or other self-paced presentation modes to investigate reading processes at the discourse level. Read\&Answer can also be easily used in combination with verbal protocols (Cerdán \& Vidal-Abarca, 2008).

\section{Validation of Read\&Answer 2.0}

In order to validate Read\&Answer 2.0, we present two types of data. First, we summarize a study in which we compared Read\&Answer and paper-and-pencil performance using two equivalent materials. Second, we present data from an experiment to compare Read\&Answer and eye movement data.

Comparison between Read\&Answer and paper-and-pencil data Martínez et al. (2009) compared performance data from a paper-and-pencil version of a comprehension test and performance on the same test administered electronically with a previous and simpler version of Read\&Answer. This version was very similar to the one presented here, except that the masking procedure was implemented by substituting letters with black balls, ${ }^{2}$ instead of the blurring procedure used in this new version. The test consisted of two expository texts, one 469 words long and the other 548 words in length, and ten multiple-choice questions per text. Questions were of four types, following Kintsch's (1998) theory of comprehension: text-based, bridging inference, global inference, and elaborative inference. Two groups of children from the 5 th, 7 th, and 9 th grades (i.e., 11, 13, and 15 years old, respectively) read the texts and answered the questions on either the paper-and-pencil version or the Read\&Answer version, being allowed to refer back to the text to search for the answer in both conditions.

Results showed that the paper-and-pencil and the electronic versions were equivalent in terms of performance scores. Additionally, both versions discriminated adequately among course levels. We only found a small difference in the homogeneity of some items, which was lower in the computer version than in the paper-and-pencil version. These slightly lower indexes were also responsible for the slightly lower reliability of the computer-based version in comparison to the paper-and-pencil version. Regarding the validity analysis, we performed a Pearson correlation

\footnotetext{
$\overline{2}$ The size of the balls fit the size of the corresponding letters (i.e., a ball substituting the letter $m$ was bigger than another substituting the letter $n$ ). This way the masking/unmasking procedure did not change the position of words in a sentence, which is important for keeping the spatial layout of the text on the screen identical across the procedure.
} 
between the total scores obtained on each version and the scores obtained on a standardized comprehension test, which yielded very similar indices. Therefore, we can state that students using Read\&Answer scored similarly to an equivalent group that used the paper-and-pencil test.

Study 2: Comparison between Read\&Answer and eye movements The previous study evaluated the similarities and differences between Read\&Answer and paper-andpencil presentation only on students' performance. In this study, we compared the on-line data provided by Read\& Answer and those obtained by a non-intrusive method like eye-tracking. We evaluated three important reading indexes: inspection time, number of visits to a segment/AOI, and reading scan paths. In the Read\&Answer condition, the inspection time index referred to the total time a segment had been unmasked, while in the eye-tracking condition it referred to the sum of all fixations on an AOI. The second index, number of visits, referred to the number of times a segment had been unmasked (Read\&Answer) or visited (eye-tracking). For these first two indexes we analyzed first-pass reading (i.e., initial visit to a segment/AOI before a participant accessed the questions for that document) and second-pass reading (i.e., look-backs to a segment/AOI before a participant accessed the questions for that document). In traditional reading studies, where students do not perform any task but just have to read and understand the text, second-pass fixations are often assumed to reflect some type of breakdown in comprehension, especially when longer regressions (e.g., look-back fixations) are considered (Rayner, Chace, Slattery, \& Ashby, 2006). Nevertheless, as we discussed in depth in the introduction, in task-oriented reading students are allowed to search the document to look for answers. Therefore, secondpass data in task-oriented reading do not necessarily reflect difficulty in text comprehension, but in some cases they may be linked to search activities. To account for this distinction, we included a third type of visit, searches, defined as the visits to a segment/AOI after a participant has accessed the questions for that document. Finally, we also compared the performance scores obtained by students with both methods.

Based on our review of prior studies, we expected that Read\&Answer would affect only the on-line variables related to the use of the mouse technology required to unmask the text segments (reading times and number of visits), but not to those reflecting the general strategy used by students (scanpath), which would be reflected in similar comprehension outcomes. We expected the following outcomes:

(1) The use of Read\&Answer will result in longer student reading times compared to eye-tracking, because in order to fixate a sentence in the first method students not only have to move their eyes to a sentence, but they also have to guide the mouse and click on the segments to unmask them (Bednarik \& Tukiainen, 2007; Jansen et al., 2003).

(2) The use of Read\&Answer will result in fewer additional visits (i.e., second pass and searches) to a particular segment/AOI compared to eye-tracking. These actions are not essential for comprehension, as is the case for first-pass visits, and their use may be related to the cost associated with them. Read\& Answer demands additional costs for revisiting a segment, i.e., those related to the controlled act of unmasking a segment with the mouse.

(3) Read\&Answer will not influence the global reading strategies used by students to move through the text and the questions (Bednarik \& Tukiainen, 2007). Thus, we expect that students' scan-paths will be similar between conditions.

(4) Based on prior results, we expect that Read\&Answer will not influence students' performance compared to the non-intrusive method of eye-tracking (study 1; Bednarik \& Tukiainen, 2007; Jansen et al., 2003).

Forty-seven university students ( $87 \%$ female) took part in the experiment. They were randomly assigned to two experimental conditions. In the first condition $(N=22)$, the texts and questions were presented on a computer screen using the Read\&Answer application. In the second condition $(N=25)$, the students performed the task using a mock version of Read\&Answer without segmentations, while their eye movements were recorded by an EyeLink II headmounted eye-tracking system. The system registered data binocularly at a rate of $500 \mathrm{~Hz}$.

Documents consisted of two continuous and two noncontinuous documents displayed on a computer. Continuous documents were formed by sentences grouped into paragraphs. They ranged from 274 to 426 words in length, and they dealt with different topics such as the language of bees and nuclear energy. In the continuous documents, the information in the Read\&Answer condition was blurred in segments composed of related nodes of information (one to three sentences). One of the non-continuous documents consisted of two X-Y graphs about climate change and one introductory paragraph. On the $x$ axis, each year was blurred independently, and the graph's legends were designed as independent segments. The rest of the graph was divided into 11 sections designed as vertical rectangles, which included the year and information from the graph related to that particular year. The first graph was divided into 19 blurred segments in all. The second non-continuous document was a tree diagram about traffic accidents. Each diagram box and footnote, as well as the introductory paragraph, was designed as an independent segment. The information in the eye-tracking condition was identical to 
the one displayed by Read\&Answer, except for the fact that the entire document was always visible to the student. Each document included three to five questions. We constructed three types of questions following the PISA framework (OECD, 2002): retrieving, interpreting, and reflectionevaluation. Retrieving questions required participants to select and extract specific pieces of information from the texts. On the other hand, interpreting questions required students to connect several pieces of information through inferences within a text. Finally, reflection-evaluation questions required participants to reflect beyond the text. Participants had to answer a total of seventeen questions. Fourteen questions were designed as multiple-choice questions with four alternatives, and three were open-ended questions.

In the Read\&Answer condition, students performed the task individually in one session in a computer classroom in groups of 5- 15 students. In the eye-tracking condition, students were assessed individually. Students first completed a general reading comprehension test in paper and pencil format (Martínez et al., 2009). Afterwards, they received an explanation about the procedure for using Read\&Answer. In the eye-tracking condition, participants were seated approximately $60 \mathrm{~cm}$ from the presentation screen. Calibration of the eye tracker was performed prior to the reading phase, and a drift-correction was done each time the participant moved to a new screen. Finally, students from both conditions were presented with the experimental task and received the following instructions: "In order to perform this task, you must use the information in the documents to answer the questions. You are free to proceed to reading either the documents or the questions first to give an answer. You are also allowed to refer back to the document to answer the question". There was no time limit, but most students finished in 20-25 min.

Prior to the analyses, we filtered the reading time data. Data from the eye-tracking condition were segmented into areas of interest (AOI) corresponding to the same segments used in the Read\&Answer condition. Then, we used the same procedure for the reading time data from both conditions. Individual distributions were analyzed to detect outliers (fixation times of $2 \mathrm{SD}$ above or below the participant's mean). These values were replaced by the participant's mean fixation time (between $1.53-2.50 \%$ of the data). Reading time data were weighted on the basis of the number of words, except for the segments corresponding to graphical information from the non-continuous document 1 (climate change). Finally, reading time data were averaged by the number of regions/AOIs in each document.

In addition, we computed the similarity of students' scan paths in each document. Eye movements were transformed into transitions between text segments. We applied the PRONET method (Cooke, Neville, \& Rowe, 1996; Rowe, Cooke, Hall, \& Halgren, 1996) to students' frequency transition matrices. This method uses the Pathfinder algorithm to simplify the original matrix, and it only retains the most salient transitions in a Pathfinder network (Schvaneveldt, 1990). Lastly, we compared each student network with the networks of the rest of the participants and obtained two indices: average similarity with students from the Read\&Answer condition and average similarity with students from the eye-tracking condition.

For each of the four documents used, we performed an ANCOVA with condition (Read\&Answer and eye-tracking) as the independent variable and general reading abilities as covariate. First, we analyzed the impact of condition on the percentage of correct responses for each document. Participants in the Read\&Answer and eye-tracking conditions scored similarly across the four documents (see Table 1 for results and statistical values).

Second, regarding the reading times analysis, we included condition (Read\&Answer and eye-tracking) and type of visit (first pass, second pass, and visits during the search) as independent variables. The analyses were performed individually for each of the four documents. In addition, in the two non-continuous documents we differentiated between textual and graphical information. Results and statistical values from the analyses performed in this section are summarized in Tables 2, 3, and 4. Whenever Mauchly's test of sphericity showed heterogeneity of covariances, the more conservative Greenhouse-Geisser test was performed. Post hoc comparisons were performed using the Spjotvoll/Stoline test for unequal sample sizes.

For the variable inspection times, condition showed significant differences across all documents. Participants using Read\&Answer spent a longer time reading the documents than those using the eye tracker. Students' inspection times did not differ according to type of visit on both non-continuous documents, but it did on both
Table 1 Performance data (percentage of correct responses) and statistical effects by condition (Read\&Answer and eye-tracking) for each of the four documents used

\begin{tabular}{llll}
\hline \multicolumn{5}{c}{ Read\&Answer } & Eye-tracking & \\
\hline Non-continuous document 1 & $0.60(0.22)$ & $0.61(0.22)$ & $\mathrm{F}(1,44)=0.03, \mathrm{p}=0.85$, partial $\eta^{2}=0.00$ \\
Non-continuous document 2 & $0.86(0.20)$ & $0.77(0.20)$ & $\mathrm{F}(1,44)=2.37, \mathrm{p}=0.13$, partial $\eta^{2}=0.05$ \\
Continuous document 1 & $0.82(0.12)$ & $0.75(0.17)$ & $\mathrm{F}(1,44)=2.13, \mathrm{p}=0.15$, partial $\eta^{2}=0.04$ \\
Continuous document 2 & $0.72(0.20)$ & $0.75(0.21)$ & $\mathrm{F}(1,44)=0.38, \mathrm{p}=0.53$, partial $\eta^{2}=0.00$ \\
\hline
\end{tabular}


Table 2 Mean inspection time (left columns) and number of visits (right columns) by condition (Read\&Answer and eye-tracking) and type of visit (first-pass, second-pass, and visits during the search) for each of the four documents used

\begin{tabular}{|c|c|c|c|c|}
\hline & \multicolumn{2}{|l|}{ Inspection time } & \multicolumn{2}{|c|}{ Number of visits } \\
\hline & Read\&Answer & Eye-tracking & Read\&Answer & Eye-tracking \\
\hline \multicolumn{5}{|c|}{ Non-continuous document 1 (text) } \\
\hline First pass & $330.75(88.62)$ & $78.75(52)$ & $0.81(0.27)$ & $0.83(0.20)$ \\
\hline Second pass & $77.00(108.50)$ & $58.5(49.62)$ & $0.23(0.33)$ & $1.63(1.56)$ \\
\hline Search & $132.37(111.37)$ & $71.00(78.37)$ & $0.60(0.57)$ & $2.86(2.70)$ \\
\hline \multicolumn{5}{|c|}{ Non-continuous document 1 (graph) } \\
\hline First pass & $1427.68(585.63)$ & $204.36(68.54)$ & $0.66(0.31)$ & $0.72(0.27)$ \\
\hline Second pass & $528.31(694.90)$ & $236.50(148.31)$ & $0.29(0.38)$ & $0.85(0.53)$ \\
\hline Search & $1225.63(818.00)$ & $450.04(313.31)$ & $0.75(0.60)$ & $1.73(1.04)$ \\
\hline \multicolumn{5}{|c|}{ Non-continuous document 2 (text) } \\
\hline First pass & $362.55(115.19)$ & $81.162(51.54)$ & $0.76(0.22)$ & $0.66(0.19)$ \\
\hline Second pass & $46.69(63.66)$ & $47.46(55.19)$ & $0.23(0.29)$ & $0.64(0.51)$ \\
\hline Search & $112.30(188.39)$ & $18.99(25.84)$ & $0.39(0.57)$ & $0.49(0.58)$ \\
\hline \multicolumn{5}{|c|}{ Non-continuous document 2 (graph) } \\
\hline First pass & $644.45(279.81)$ & $266.90(180.54)$ & $0.92(0.23)$ & $0.96(0.14)$ \\
\hline Second pass & $197.18(209.36)$ & $412.36(354.18)$ & $0.55(0.58)$ & $2.26(1.39)$ \\
\hline Search & $570.00(386.72)$ & $327.27(251.81)$ & $1.45(1.11)$ & $2.62(2.02)$ \\
\hline \multicolumn{5}{|c|}{ Continuous document 1} \\
\hline First pass & $363.33(94.33)$ & $170.44(60.24)$ & $0.90(0.18)$ & $0.92(0.06)$ \\
\hline Second pass & $43.60(40.33)$ & $78.46(80.93)$ & $0.14(0.16)$ & $0.95(1.17)$ \\
\hline Search & $122.20(126.60)$ & $54.53(69.26)$ & $0.67(0.79)$ & $1.34(1.44)$ \\
\hline \multicolumn{5}{|c|}{ Continuous document 2} \\
\hline First pass & $411.86(123.46)$ & $164.33(37.26)$ & $0.82(0.20)$ & $0.86(0.09)$ \\
\hline Second pass & $41.64(45.40)$ & $49.86(54.80)$ & $0.20(0.21)$ & $0.55(0.54)$ \\
\hline Search & $122.46(129.86)$ & $59.06(68.13)$ & $0.89(0.96)$ & $1.36(1.22)$ \\
\hline
\end{tabular}

Inspection times correspond to milliseconds per word by segments/AOIs, except for the graphical zones of non-continuous documents 1 , in which time data were not weighed. Number of visits was averaged by the number of segments/AOI in each document. Standard deviations are presented in brackets

continuous documents: first-pass inspection time was higher than second-pass and search times. In addition, condition significantly interacted with type of visit across all documents. Post hoc comparisons revealed two different patterns. On the one hand, in the graphical zones of the two non-continuous documents and in continuous document 1 (bee's language), students using Read\&Answer spent more time on first passes and searches than those using the eye tracker, and none of the differences approached significance levels for the second-pass time. On the other hand, in the textual zones of the two non-continuous documents and in continuous document 2 (nuclear energy), students using Read\&Answer spent more time than those using the eye tracker only on first-pass visits. Differences in second-pass and search times did not prove significant. Globally, firstpass inspection times were longer in Read\&Answer, whereas most second-pass and search times were similar to those obtained with the eye tracker, which can be explained by the cost of clicking a segment with a mouse to read a piece of text, especially during the first pass.

The analyses of number of visits revealed significant effects for condition. Across all four documents, participants in the eye-tracking condition performed more visits than those using Read\&Answer. The effect of type of visit was not significant, except on continuous document 1 (bee's language), where students performed fewer secondpass visits than first-pass and visits during the search. Condition significantly interacted with type of visit across all documents (except for continuous document 2). Post hoc comparisons showed that participants using the eye tracker performed more second passes and visits during the search than the students using Read\&Answer, but had similar firstpass visits (differences in continuous document 2, although not significant, showed a similar pattern). In sum, taking together the data of inspection times and number of visits, we can conclude that students using Read\&Answer 
Table 3 Statistical effects for inspection times (time by word) by condition (Read\&Answer and Eye-tracking) and type of visit (first-pass, second-pass, and visits during the search) for each of the four documents used

\begin{tabular}{|c|c|c|c|}
\hline & Type of visit & Condition & Interaction \\
\hline Non-continuous document 1 (text) & $\begin{array}{l}F(2,88)=0.20, p=0.81 \\
\text { partial } \eta^{2}=0.00\end{array}$ & $\begin{array}{l}F(1,44)=48.41, p=0.00 \\
\quad \text { partial } \eta^{2}=0.52\end{array}$ & $\begin{array}{l}F(2,88)=27.13, p=0.00 \\
\quad \text { partial } \eta^{2}=0.38\end{array}$ \\
\hline Non-continuous document 1 (graph) & $\begin{array}{l}F(1.77,78.06)=0.67, p=0.49 \\
\quad \text { partial } \eta^{2}=0.01\end{array}$ & $\begin{array}{l}F(1,44)=66.72, p=0.00 \\
\quad \text { partial } \eta^{2}=0.60\end{array}$ & $\begin{array}{l}F(1.77,78.06)=10.44, p=0.00, \\
\quad \text { partial } \eta^{2}=0.19\end{array}$ \\
\hline Non-continuous document 2 (text) & $\begin{array}{l}F(2,88)=1.78, p=0.18 \\
\text { partial } \eta^{2}=0.03\end{array}$ & $\begin{array}{l}F(1,44)=44.13, p=0.00 \\
\quad \text { partial } \eta^{2}=0.50\end{array}$ & $\begin{array}{l}F(2,88)=31.40, p=0.00 \\
\quad \text { partial } \eta^{2}=0.41\end{array}$ \\
\hline Non-continuous document 2 (graph) & $\begin{array}{l}F(1.75,77.31)=0.38, p=0.68 \\
\quad \text { partial } \eta^{2}=0.00\end{array}$ & $\begin{array}{l}F(1,44)=8.54, p=0.00 \\
\quad \text { partial } \eta^{2}=0.16\end{array}$ & $\begin{array}{l}F(1.75,77.31)=12.77, p=0.00 \\
\quad \text { partial } \eta^{2}=0.22\end{array}$ \\
\hline Continuous document 1 & $\begin{array}{l}F(2,88)=2.65, p=0.06 \\
\text { partial } \eta^{2}=0.06\end{array}$ & $\begin{array}{l}F(1,44)=42.06, p=0.00 \\
\text { partial } \eta^{2}=0.48\end{array}$ & $\begin{array}{l}F(2,88)=19.99, p=0.00 \\
\quad \text { partial } \eta^{2}=0.31\end{array}$ \\
\hline Continuous document 2 & $\begin{array}{l}F(1.69,74.76)=3.66, p=0.04 \\
\quad \text { partial } \eta^{2}=0.07\end{array}$ & $\begin{array}{l}F(1,44)=43.24, p=0.00 \\
\quad \text { partial } \eta^{2}=0.49\end{array}$ & $\begin{array}{l}F(1.69,74.76)=31.18, p=0.00 \\
\quad \text { partial } \eta^{2}=0.41\end{array}$ \\
\hline
\end{tabular}

performed longer but fewer revisits and visits during the search, whereas those using the eye-tracking performed shorter and more revisits and visits, which can be interpreted as a sort of trade-off between the cost of re-reading (either simply looking back or using the mouse as a tool) and the need to re-process text information.

Finally, an inspection of average scan-path matrices provided by the Pathfinder algorithm for each condition revealed that students from the Read\&Answer condition performed fewer significant backward transitions in continuous documents $(\mathrm{M}=1.5)$ than those from the eye-tracking condition $(\mathrm{M}=9.5)$, but similar significant forward transitions (for both conditions $\mathrm{M}=15$ ). With regard to non-continuous documents, average matrices for the diagram document showed that students from the two conditions did not differ on relevant transitions, as identified in previous studies (Salmerón, Cañas, Kintsch \& Fajardo, 2005), such as the number of transitions between linked nodes in the diagram document (10 and 9 transitions for the Read\&Answer and eye-tracking conditions, respectively) or between non linked nodes (4 and 3 transitions). Similarly, average matrices for the X-Y graphs did not differ in the number of transitions between the content of the graph to the graph's title, axis, or legend, and vice versa (15 and 13 transitions for the Read\&Answer and eye-tracking conditions, respectively), which correspond to significant transitions of undergraduate students (Carpenter \& Shah, 1998). In sum, relevant student transitions between text segments were similar across conditions. The higher number of backward transitions in the eye-tracking condition may simply be related to the higher number of secondpass visits performed by students in this condition.

To sum up, the global pattern of results concur with prior research in that the masking/blurring procedure does not interfere with normal reading strategies and performance, but it has an impact on some on-line measures. Compre-

Table 4 Statistical effects for number of visits by condition (Read\&Answer and eye-tracking) and type of visit (first pass, second pass, and visits during the search) for each of the four documents used

\begin{tabular}{lccc}
\hline & Type of visit & Condition & Interaction \\
\hline $\begin{array}{l}\text { Non-continuous document } 1 \\
\text { (text) }\end{array}$ & $F(1.31,57.87)=0.30, p=0.64$, & $F(1,44)=32.29, p=0.00$, & $F(1.31,57.87)=8.60, p=0.00$, \\
partial $\eta^{2}=0.00$ & partial $\eta^{2}=0.42$ & $F(1.14,50.45)=6.72, p=0.01$, \\
Non-continuous document 1 & $F(1.14,50.45)=1.65, p=0.20$, & $F(1,44)=28.48, p=0.00$, & partial $\eta^{2}=0.13$ \\
(graph) & partial $\eta^{2}=0.03$ & partial $\eta^{2}=0.39$ & $F(1.47,64.75)=4.02, p=0.03$, \\
Non-continuous document 2 & $F(1.47,64.75)=0.15, p=0.78$, & $F(1,44)=2.90, p=0.09$, & partial $\eta^{2}=0.08$ \\
(text) & partial $\eta^{2}=0.00$ & partial $\eta^{2}=0.06$ & $F(1.43,63.22)=6.17, p=0.00$, \\
Non-continuous document 2 & $F(1.43,63.22)=0.82, p=0.44$, & $F(1,44)=23.78, p=0.00$, & partial $\eta^{2}=0.12$ \\
(graph) & partial $\eta^{2}=0.01$ & partial $\eta^{2}=0.35$ & $F(1.57,69.15)=2.93, p=0.07$, \\
Continuous document 1 & $F(1.57,69.15)=8.76, p=0.00$, & $\mathrm{F}(1,44)=11.38, p=0.00$, & partial $\eta^{2}=0.06$ \\
& partial $\eta^{2}=0.16$ & partial $\eta^{2}=0.20$ & $F(1.19,52.54)=1.13, p=0.30$, \\
Continuous document 2 & $F(1.19,52.54)=0.81, p=0.44$, & $F(1,44)=5.79, p=0.02$, & partial $\eta^{2}=0.02$ \\
& partial $\eta^{2}=0.01$ & partial $\eta^{2}=0.12$ & \\
\hline
\end{tabular}


hension scores did not differ between students using Read\&Answer or eye-tracking (Bednarik \& Tukiainen, 2007; Jansen, et al., 2003), which is consistent with the fact that students used similar global reading strategies under the two systems. Thus, the results show that the burden imposed by Read\&Answer, related to using the computer mouse to make the text segments visible, did not critically affect students' reading performance. Nevertheless, Read\&Answer had an impact on some on-line measures more sensitive to the use of the mouse technology to unmask text segments. In this sense, participants using Read\&Answer spent more time reading the documents, which concurs with prior studies using comparable masking procedures (Bednarik \& Tukiainen, 2007; Jansen, et al., 2003). This difference may be due in part to the extra time required to guide the mouse cursor to the next segment, as has been suggested in previous studies using mousecontrolled text access.

\section{Conclusions}

Read\&Answer is a useful tool for studying online processes in task-oriented reading activities. It mimics paper-andpencil scenarios where a reader has to read one or more documents to do different tasks, such as answering questions, writing an essay, taking notes, and so on. It presents documents and tasks in a masked way. The reader unmasks a piece of a document or the task by clicking on it with a mouse. Then, the computer records any action in milliseconds, and a different application called RADAR analyzes and summarizes the raw data after the researcher creates some variables.

Previous versions of Read\&Answer have shown their utility in investigating theoretical and applied issues regarding online processes in task-oriented reading, such as the role of task variables in explaining comprehension and learning results (Cerdán \& Vidal-Abarca, 2008; Cerdán et al., 2009; Gil et al., in press) or individual differences in self-regulating the interaction between the reader and the text when answering questions from a text (Vidal-Abarca et al., in press). Read\&Answer has also been successfully used to develop a comprehension test that records on-line data and combines them with performance scores to provide practitioners with an automatic assessment of the reader's strategies (Martínez,et al., 2009). Read\&Answer can also be used to investigate other theoretical and practical issues at the discourse level, such as the relevance effect (McCrudden \& Schraw, 2007).

The comparison of Read\&Answer and eye movements shows that performance level is not impaired by the potential constraints introduced by Read\&Answer, such as the need to use the mouse or the masking procedure.
However, Read\&Answer has an impact on some on-line measures more sensitive to the use of the mouse technology to unmask text segments. Nevertheless, this impact does not alter the strategic interaction between the reader and the text in task-oriented reading activities.

Authors' Note The research was supported by the Secretaría de Estado de Política Científica y Tecnológica (SEPCT) of the Spanish Ministry of Education (SME), Grant SEJ2005-04500. Any opinions, findings, conclusions, or recommendations expressed in this article are those of the authors and do not necessarily reflect the views of the SEPCT.

\section{References}

Bednarik, R., \& Tukiainen, M. (2007). Validating the restricted focus viewer: A study using eye-movement tracking. Behavior Research Methods, 39(2), 274-282.

Carpenter, P., \& Shah, P. (1998). A model of the perceptual and conceptual processes in graph comprehension. Journal of Experimental Psychology. Applied, 4(2), 75-100.

Cerdán, R., \& Vidal-Abarca, E. (2008). The effects of tasks on integrating information from multiple documents. Journal of Educational Psychology, 100(1), 209-222.

Cerdán, R., Vidal-Abarca, E., Martínez, M., Gilabert, R., \& Gil, L. (2009). Impact of question-answering tasks on search processes and reading comprehension. Learning and Instruction, 19, 13-27.

Cooke, N. J., Neville, K. J., \& Rowe, A. L. (1996). Procedural network representations of sequential data. Human-Computer Interaction, 11, 29-68.

Coté, N., Goldman, S. R., \& Saul, E. U. (1998). Students making sense of informational text: Relation between processing and representations. Discourse Processes, 25(1), 1-53.

Duggan, G. B., \& Payne, S. J. (2009). Text skimming: The process and effectiveness of foraging through text under time pressure. Journal of Experimental Psychology. Applied, 15, 228-242.

Ferrer, A., Vidal-Abarca, E., Ávila, V., Mañá, A., \& Llorens, A. C. (2010). The role of question format and text availability on Reading Comprehension Assessment. 1st joint conference of the Experimental Psychology Society (EPS) and the Spanish Experimental Psychological Society. Granada, Spain.

Gil, L., Braten, I., Vidal-Abarca, E., \& Stromso, H. I. (in press). Summary versus Argument Tasks when Working with Multiple Documents: Which is Better for Whom? Contemporary Educational Psychology. doi: 10.1016/j.cedpsych. 2009.11.002

Gil, L., Vidal-Abarca, E., \& Martínez, T. (2008). Eficacia de tomar notas para integrar información de varios textos (Efficacy of note-taking to integrate information from multiple documents). Infancia y Aprendizaje, 31(2), 259-272.

Goldman, S. R., \& Saul, E. U. (1990a). Applications for tracking reading behavior on the Macintosh. Behavior Research Methods, Instruments, \& Computers, 22(6), 526-532.

Goldman, S. R., \& Saul, E. U. (1990b). Flexibility in text processing: A strategy competition model. Learning and Individual Differences, 2, 181-219.

Hyönä, J., Lorch, R. F., \& Kaakinen, J. K. (2002). Individual differences in reading to summarize expository text: Evidence From eye fixation patterns. Journal of Educational Psychology, 94(1), 44-55. 
Jansen, A. R., Blackwell, A. F., \& Marriot, K. (2003). A tool for tracking visual attention: The Restricted Focus Viewer. Behavior Research Methods, Instruments, \& Computers, 35 (1), 57-69.

Just, M. A., \& Carpenter, P. A. (1980). A theory of reading: From eye fixations to comprehension. Psychological Review, 87, 329-354.

Kaakinen, J. K., \& Hyönä, J. (2005). Perspective effects on expository text comprehension: Evidence from think-aloud protocols, eyetracking, and recall. Discourse Processes, 40(3), 239-257.

Kaakinen, J. K., Hyönä, J., \& Keenan, J. M. (2003). How prior knowledge, WMC, and relevance of information affect eye fixations in expository text. Journal of Experimental Psychology. Learning, Memory, and Cognition, 29, 447-457.

Kintsch, W. (1998). Comprehension. A paradigm for cognition. Cambridge: Cambridge University Press.

Maña, A., Vidal-Abarca, E., Dominguez, C., Gil, L., \& Cerdán, R. (2009). Papel de los procesos metacognitivos en una tarea de pregunta-respuesta con textos escritos (The role of metacognitive processes on a question-answering task with written texts). Infancia y Aprendizaje, 32(4), 553-565.

Martínez, T., Vidal-Abarca, E., Gil, L., \& Gilaber, R. (2009). On-line assessment of comprehension processes. The Spanish Journal of Psychology, 12, 308-319.

McConkie, G. W., \& Rayner, K. (1975). The span of the effective stimulus during a fixation in reading. Perception \& Psychophysics, 17(6), 578-586.

McCrudden, M. T., Magliano, J., \& Schraw, G. (2009). Exploring how relevance instructions affect personal reading intentions, reading goals, and text processing: A mixed methods study. Contemporary Educational Psychology. Advance online publication. doi:10.1016/ j.cedpsych.2009.12.001

McCrudden, M. T., \& Schraw, G. (2007). Relevance and goalfocusing in text processing. Educational Psychology Review, 19, $113-139$.

OECD. (2002). Programme for international student assessment: Sample tasks from the PISA 2000 assessment of reading, mathematical and scientific literacy. Paris: OECD.
Ozuru, Y., Best, R., Bell, C., Witherspoon, A., \& McNamara, D. (2007). Influence of question format and text availability on the assessment of expository text comprehension. Cognition and Instruction, 25(4), 399-438.

Rayner, K. (1998). Eye movements in reading and information processing: 20 years of research. Psychological Bulletin, 124 (3), 372-422.

Rayner, K., Chace, K., Slattery, T., \& Ashby, J. (2006). Eye movements as reflections of comprehension processes in reading. Scientific Studies of Reading, 10(3), 241-255.

Rouet, J-F. (2006). Question answering and document search. In JF. Rouet (Ed.), The skills of document use. From text comprehension to web-based learning (pp. 93-121). Mahwah, NJ: Erlbaum.

Rouet, J.-F., \& Passerault, J.-M. (1999). Analyzing learnerhypermedia interaction: An overview of on-line methods. Instructional Science, 27, 201-219.

Rouet, J.-F., Vidal-Abarca, E., Bert-Erboul, A., \& Millogo, V. (2001). Effects of information search tasks on the comprehension of instructional text. Discourse Processes, 31, 163-186.

Rowe, A. L., Cooke, N. J., Hall, E. P., \& Halgren, T. L. (1996). Toward an on-line knowledge assessment methodology: Building on the relationship between knowing and doing. Journal of Experimental Psychology. Applied, 2, 31-47.

Salmerón, L., Cañas, J. J., Kintsch, W., \& Fajardo, I. (2005). Reading strategies and hypertext comprehension. Discourse Processes, 40, 171-191.

Salmerón, L., Vidal-Abarca, E., Mana, A., Martínez, T., Gil, L., \& Naumann, J. (2010). Reading strategies in task oriented reading: The case of PISA-like tasks. Manuscript submitted for publication

Schvaneveldt, R. (Ed.). (1990). Pathfinder associative networks: Studies in knowledge organization. Norwood: Ablex.

Snow, C. \& RAND Reading Study Group (2002). Reading for understanding. RAND URL: http://www.rand.org/

Vidal-Abarca, JE., Mañá, A. \& Gil, L. (in press). Individual differences for self-regulating task-oriented reading activities. Journal of Educational Psychology. doi:10.1037/a0020062 